\title{
Mindfulness no contexto dos transtornos mentais: uma revisão integrativa*
}

Paula Hayasi Pinho

(D) https://orcid.org/0000-0001-8922-0699

Lígia Maffei Carnevalli ${ }^{1}$

(D) https://orcid.org/0000-0002-2139-2918

Renata Oliveira Santos ${ }^{1}$

(D) https://orcid.org/0000-0002-3865-1496

Luanna Carolyne Silva De Lacerda ${ }^{1}$

(D) https://orcid.org/0000-0003-3788-9029

* Este artigo refere-se à chamada temática "Mindfulness e outras práticas contemplativas".

1 Universidade Federal do Recôncavo da Bahia, Centro de Ciências da Saúde, Santo Antônio de Jesus, BA, Brasil.

\begin{abstract}
Objetivo: analisar as evidências de como a prática das intervenções baseadas em mindfulness tem sido empregada terapeuticamente e quais os efeitos dessas intervenções em adultos e idosos acometidos por transtornos mentais. Método: revisão integrativa de artigos publicados entre 2008 a 2018, nas bases: Biblioteca Virtual em Saúde, Scientific Electronic Library Online e PubMed. Após aplicação dos critérios de inclusão e exclusão e da metodologia PRISMA foram selecionados 37 artigos a serem analisados. Resultados: encontrou-se variabilidade nos métodos das intervenções baseadas em mindfulness, sendo Redução de Estresse Baseado em Mindfulness (MBSR), Terapia Cognitiva Baseada em Mindfulness (MBCT) e, a Prevenção à Recaída Baseada em Mindfulness (MBRP) as mais utilizadas. As práticas de intervenções baseadas no mindfulness apresentaram benefícios fisiológicos, melhora no bem-estar psicológico e emocional e mostraram-se ser moduladoras de interação social. Influências positivas foram observadas no gerenciamento do estresse, na redução do grau de ansiedade e sintomas de depressão e no desenvolvimento da atenção. Achados promissores foram encontrados sobre o uso dessas práticas em indivíduos com sintomas psicóticos. Conclusão: sugere-se que a prática de intervenções baseadas em mindfulness desenvolve habilidades que auxiliam no processo terapêutico de diversos transtornos mentais.
\end{abstract}

Descritores: Atenção Plena; Terapêuticas; Transtornos Mentais; Saúde Mental; Adulto; Idoso.

\section{Como citar este artigo}

Pinho PH, Carnevalli LM, Santos RO, Lacerda LCS. Mindfulness-based interventions for adults and older adults with mental disorders: An integrative review. SMAD, Rev Eletrônica Saúde Mental Álcool Drog. 2020;16(3):105-117. doi: https://dx.doi.org/10.11606/issn.1806-6976.smad.2020.166027 


\section{Mindfulness-based interventions for adults and older adults with mental disorders: an integrative review}

Objective: to analyze the evidence of how the practice of mindfulness-based interventions has been used therapeutically and what are the effects of these interventions on adults and the elderly affected by mental disorders. Method: integrative review of articles published between 2008 and 2018, based on: Virtual Health Library, Scientific Electronic Library Online and PubMed. After applying the inclusion and exclusion criteria and the PRISMA methodology, 37 articles were selected to be analyzed. Results: variability was found in the methods of mindfulness-based interventions, with Mindfulness-Based Stress Reduction (MBSR), Mindfulness-Based Cognitive Therapy (MBCT) and Mindfulness-Based Relapse Prevention (MBRP) the most widely used. The practices of mindfulness-based interventions have shown physiological benefits, improved psychological and emotional well-being and have been shown to modulate social interaction. Positive influences were observed in the management of stress, in the reduction of the degree of anxiety and symptoms of depression and in the development of attention. Promising findings have been found on the use of these practices in individuals with psychotic symptoms. Conclusion: it is suggested that the practice of mindfulness-based interventions develops skills that assist in the therapeutic process of various mental disorders.

Descriptors: Mindfulness; Therapeutics; Mental Disorders; Mental Health; Adult; Aged.

\section{Intervenciones basadas en mindfulness para adultos y ancianos con trastornos mentales: una revisión integradora}

Objetivo: analizar la evidencia de cómo la práctica de las intervenciones basadas en la atención plena se ha utilizado terapéuticamente y cuáles son los efectos de estas intervenciones en adultos y ancianos afectados por trastornos mentales. Método: revisión integradora de artículos publicados entre 2008 y 2018, basada en: Biblioteca Virtual en Salud, Biblioteca Electrónica Científica en línea y PubMed. Después de aplicar los criterios de inclusión y exclusión y la metodología PRISMA, se seleccionaron 37 artículos para ser analizados. Resultados: se encontró variabilidad en los métodos de intervenciones basadas en la atención plena, con la reducción del estrés basada en la atención plena (MBSR), la terapia cognitiva basada en la atención plena (MBCT) y la prevención de la recaída basada en la atención plena (MBRP) las más utilizadas. Las prácticas de las intervenciones basadas en la atención plena han mostrado beneficios fisiológicos, un mejor bienestar psicológico y emocional y han demostrado que modulan la interacción social. Se observaron influencias positivas en el manejo del estrés, en la reducción del grado de ansiedad y síntomas de depresión y en el desarrollo de la atención. Se han encontrado resultados prometedores sobre el uso de estas prácticas en individuos con síntomas psicóticos. Conclusión: se sugiere que la práctica de intervenciones basadas en la atención plena desarrolle habilidades que ayuden en el proceso terapéutico de varios trastornos mentales.

Descriptores: Atención Plena; Terapéutica; Trastornos Mentales; Salud Mental; Adultos; Anciano. 


\section{Introdução}

No contexto clínico de saúde, a meditação foi trazida aos EUA no final da década de 1970 por Kabat-Zinn, que criou um programa de 8 a 10 semanas inicialmente para aqueles que sofriam de dores crônicas, sem possibilidades médicas ${ }^{(1)}$. Posteriormente, o programa foi expandido para indivíduos ansiosos e obteve resultados promissores para a redução da ansiedade e depressão(1). Esse programa, naquela época, foi intitulado "Terapia para Redução do Estresse Baseada em Mindfulness" (MBSR), e é modelo para diversas adaptações feitas em pesquisas.

Importante destacar que mindfulness, ao ser traduzido para o português, é sinônimo de atenção plena. E praticá-la é estar atento de forma consciente e intencional ao momento presente e à vivência do momento, sem se sentir absorvido por ela(2).

Treinar mindfulness ou atenção plena desenvolve a habilidade de ser menos reativo aos acontecimentos cotidianos, entretanto, requer prática regular para a manutenção dos benefícios ${ }^{(3)}$. As práticas do mindfulness são divididas em formal e informal(3). A meditação refere-se à prática formal, que permite o treino da atenção através da introspecção disciplinada e da vivência do mindfulness em níveis aprofundados, por meio da respiração ou dos exercícios de movimento relaxamento muscular progressivo consciente. Já a prática informal ocorre através da aplicação das habilidades de mindfulness na vida diária(3), ou seja, direcionada à escuta de sons do ambiente, à descrição das emoções ou percepção de sensações corporais, ou ao gosto e textura dos alimentos, por exemplo.

Dentre as doenças crônicas, e ocupando grande espaço em relação à prevalência mundial, encontram-se os transtornos mentais (TM), de etiologia múltipla e que podem afetar indivíduos independentemente do sexo, gênero e classe social. Nesse contexto, o efeito terapêutico do mindfulness tem sido positivo para depressão(4), ansiedade generalizada(5), ansiedade social, uso abusivo de álcool e outras drogas ${ }^{(6)}$, estresse póstraumático, entre outras.

Altas taxas de incapacidade e mortalidade entre pessoas com TM são evidenciadas a partir de um estudo(7) $^{(7)}$ so o peso global de doenças, lesões e fatores de risco (GBD). Nas Américas, estimativas do GBD apontam para o uso abusivo de álcool e o suicídio como principais causas de deficiência e mortalidade ${ }^{(7)}$. No Brasil, segundo dados da Organização Mundial da Saúde ${ }^{(8)}$, estima-se que para cada 100 pessoas, 30 podem vir a desenvolver problemas de saúde mental, como a depressão $(5,8 \%)$ e a ansiedade $(9,3 \%)$.

Nesse sentido, a partir da percebida necessidade de práticas auxiliares àqueles em sofrimento psíquico, contrapondo-se a práticas hegemônicas indutoras da medicalização da vida, objetivou-se realizar uma revisão integrativa da literatura para analisar as evidências de como a prática das intervenções baseadas em mindfulness tem sido empregada terapeuticamente e quais os efeitos dessas intervenções em adultos e idosos acometidos por transtornos mentais.

\section{Método}

Trata-se de uma revisão integrativa de literatura em que pode-se utilizar tanto elementos teóricos quanto empíricos $^{(9)}$. As etapas condutoras dessa revisão foram: identificação do tema e seleção da questão de pesquisa; estabelecimento dos critérios de inclusão e exclusão para coleta de dados; identificação dos estudos préselecionados e selecionados e categorização dos estudos selecionados; avaliação dos estudos incluídos na revisão integrativa; análise e interpretação dos resultados e apresentação da revisão/síntese do conhecimento(10).

Buscou-se responder à questão de pesquisa "qual a produção científica acerca da prática do mindfulness em adultos e idosos com algum quadro de transtorno mental?", elaborada por meio da estratégia "População/ Problema, Interesse e Contexto" (PICo). Estratégia baseada na segmentação da pergunta de pesquisa permitindo que o pesquisador selecione palavras que tragam a definição apropriada ao questionamento inicial, identificando a melhor informação científica acerca do tema ${ }^{(11)}$.

A coleta de dados ocorreu em agosto de 2019. Para a seleção desses artigos foram utilizadas as seguintes bases de dados: Biblioteca Virtual em Saúde (BVS), Scientific Electronic Library Online (SCIELO) e PubMed. Os descritores em ciências da saúde (DeCS) utilizados e combinados foram: "mindfulness" AND "mental health".

Foram incluídos estudos originais e revisões de literatura escritos na forma de artigo eletrônico, entre os anos de 2008 a 2018, nos idiomas português, inglês e espanhol. Foram excluídos: teses, dissertações, editoriais, materiais não científicos, artigos em que não se identificou relação com a temática, duplicados, ou que não abordassem a população adulto e idoso.

Duas revisoras realizaram a busca e seleção dos artigos de forma independente, conferindo maior rigor a este procedimento. Inicialmente, os estudos foram selecionados por meio da leitura dos títulos e resumos com base nos critérios de inclusão e exclusão. A partir dessa seleção, os artigos restantes foram lidos na íntegra, com o objetivo de incluir apenas as publicações relevantes e pertinentes ao problema deste estudo. Na Figura 1 é apresentado o processo de seleção dos estudos elaborado conforme o protocolo Preferred Reporting Items for Systematic Reviews and MetaAnalyses $^{(12)}$ (PRISMA). 


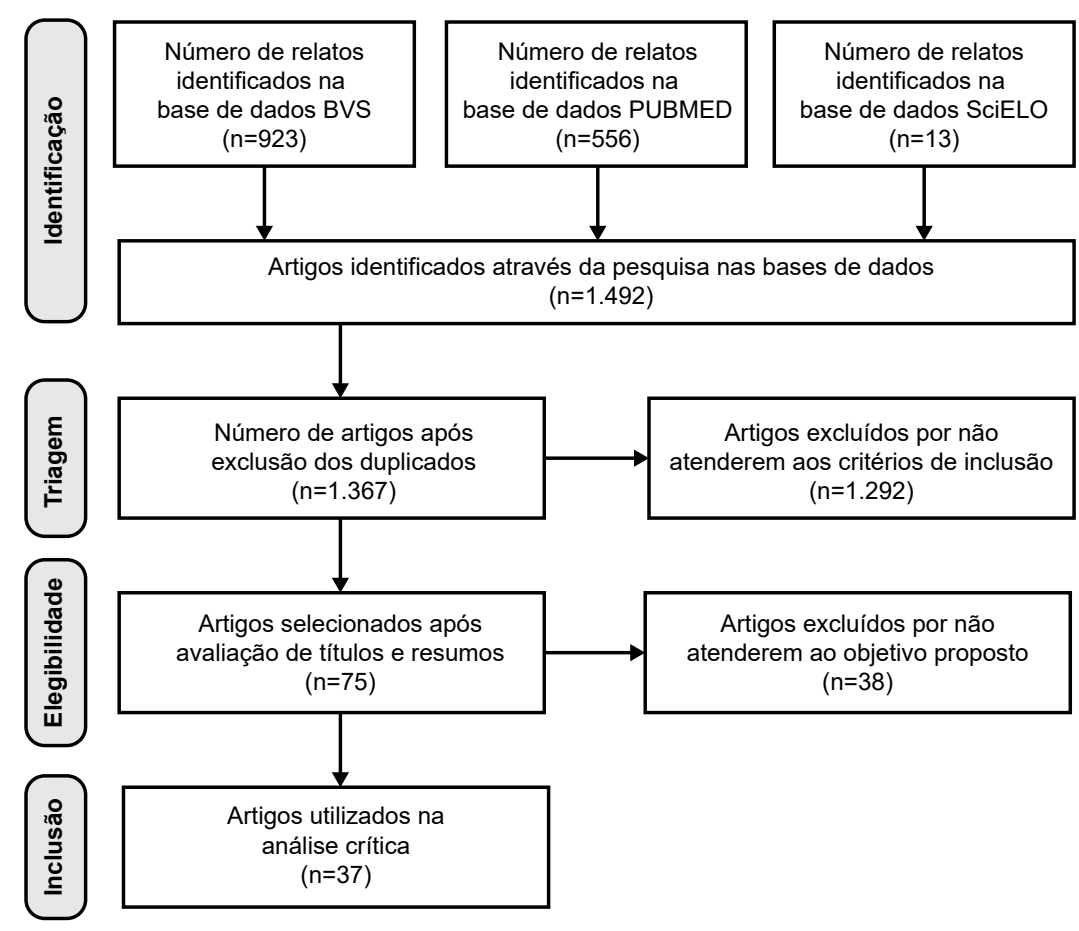

Figura 1 - Fluxograma das etapas de identificação, triagem, elegibilidade e inclusão dos artigos conforme PRISMA Flow Diagram. Santo Antônio de Jesus, Bahia, Brasil, dezembro 2019

O corpus de análise compõe-se de 37 estudos, os quais foram submetidos à nova leitura para extração de informações relevantes, considerando a pergunta norteadora deste estudo. Para organização dos dados coletados, as pesquisadoras elaboraram um instrumento incluindo as seguintes informações: autores, ano de publicação, idioma, local e tipo de estudo.

Nesta revisão, o tipo de estudo/nível de evidência foi classificado em: nível I: revisão sistemática ou metaanálise de ensaios clínicos randomizados controlados ou provenientes de diretrizes clínicas baseadas em revisões sistemáticas de ensaios clínicos randomizados controlados; nível II: pelo menos um ensaio clínico randomizado controlado bem delineado; nível III: ensaios clínicos bem delineados sem randomização; nível IV: estudos de coorte ou caso-controle bem delineados; nível V: revisão sistemática de estudos descritivos e qualitativos; nível VI: um único estudo descritivo ou qualitativo; nível VII: opinião de autoridades e/ou relatório de comitês de especialistas ${ }^{(13)}$.

\section{Resultados}

A partir da pesquisa nas plataformas online, foram encontrados 1492 artigos. Destes, seguindo a metodologia PRISMA, 37 foram selecionados para compor o banco de dados dessa revisão integrativa. Os estudos foram publicados como artigos originais $(89,1 \%)$, artigos de revisão $(5,4 \%)$, relato de caso $(2,7 \%)$ e metanálise (2,7\%) entre 2008 e 2018, sendo o ano de 2016 aquele com maior quantidade de publicações $(35,1 \%)$. O local onde os estudos foram realizados variou majoritariamente entre Estados Unidos da América (48,6\%), Reino Unido (13,5\%), e Austrália $(8,1 \%)$. Todos foram publicados em inglês (Figura 2 ).

Conforme a sistematização de níveis de evidências ${ }^{(13)}$, o maior e menor quantitativo de artigos foi classificado como nível VI ${ }^{(19,21,23-24,28-30,35-37,40,42,45,48)}$ $(37,8 \%)$ e $\mathrm{I}^{(25,49)}(5,4 \%)$, respectivamente; nenhum dos 37 artigos foram classificados como níveis V e VII; $21,6 \%$ corresponderam ao nível II ${ }^{(20,27,32,38-39,44,46-47)} ; 18,9 \%$ ao nível $\operatorname{III}^{(15,17,22,24,33-34,43)}$ e $16,2 \%$ ao $\operatorname{IV}^{(14,16,18,31,41,50)}$. Quanto ao delineamento dos estudos, 43,2\% apresentam característica quantitativa(15-18,20,26-27,32-34,38,41,43-44,46-47), $40,5 \%$ qualitativa $(19,21,23-25,28-29,35-37,40,42,48-49)$ e $16,2 \%$ quanti-quali ${ }^{(14,22,30-31,39-50)}$.

O público alvo dos estudos, previamente estabelecido como adultos e idosos (indivíduos acima de 18 anos de idade), apresentou variação entre estudantes de graduação e/ou pós-graduação(14,16-18,22,32,34,37,41), profissionais da saúde ${ }^{(29,31,35)}$, equipes de saúde mental ${ }^{(23,30)}$, entre outros.

A prática das intervenções baseadas em Mindfulness em adultos e idosos com algum quadro de TM foi explorada sob diferentes aspectos nos artigos. Além de variações na forma de aplicação da técnica, os tipos de TM apresentados pelos participantes dos estudos também são variados, inclusive aqueles derivados de e/ ou agravados em ambientes de trabalho.

A partir dos temas centrais identificados nos estudos revisados, quatro categorias temáticas emergiram: (1) Tipos de intervenções em 
mindfulness ${ }^{(14,18-20,23-24,30-31,36-40,42-43,45-49)}$; (2) Mindfulness e transtornos mentais ${ }^{(14-16,18-28,30-32,34-50)}$; (3) Mindfulness e o ambiente de trabalho(14,17,23,29,31,34-35); e (4) Benefícios

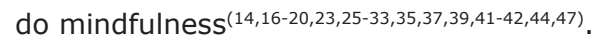

Na categoria "Tipos de intervenções em mindfulness" encontram-se estudos que exploram sobre a metodologia e princípios de aplicação do mindfulness. Na categoria "Mindfulness e transtornos mentais" são abordados TM apresentados pelos participantes dos estudos, buscando relacionar possíveis efeitos da prática de mindfulness nos comportamentos e sintomas característicos. Ainda nesse contexto, em "Mindfulness e o ambiente de trabalho" estão estudos que abrangem intervenções de mindfulness nas organizações/corporações a fim de prevenir o esgotamento profissional e/ou atenuá-los. Por fim, estudos referentes aos efeitos percebidos a partir da prática de mindfulness em esferas fisiológicas, psicoemocionais, sociais, físicas e/ou ambientais, foram reunidas na categoria "Benefícios do Mindfulness".

Dentre os tipos de intervenções baseadas em mindfulness (MBIs) notou-se variedade de metodologias e finalidades de aplicação da técnica, e distinções como: se as intervenções ocorrem em grupo ou individualmente, e de maneira presencial ou online.

\begin{tabular}{|c|c|c|c|c|}
\hline Autor(es) & Ano & Idioma & Local & Tipo de Estudo \\
\hline Aherne et al..$^{(14)}$ & 2016 & Inglês & Irlanda & Artigo Original \\
\hline Antonova et al. ${ }^{(15)}$ & 2016 & Inglês & Reino Unido & Artigo Original \\
\hline Beck et al. ${ }^{(16)}$ & 2017 & Inglês & EUA & Artigo Original \\
\hline Bentley et al. ${ }^{(17)}$ & 2018 & Inglês & EUA & Artigo Original \\
\hline Bergen-Cico et al. ${ }^{(18)}$ & 2015 & Inglês & Suécia & Artigo Original \\
\hline Berk et al. ${ }^{(19)}$ & 2018 & Inglês & EUA & Artigo Original \\
\hline Boettcher et al..$^{(20)}$ & 2014 & Inglês & EUA & Artigo Original \\
\hline Brewer et al.(21) & 2013 & Inglês & EUA & Artigo Original \\
\hline Buitron et al. ${ }^{(22)}$ & 2016 & Inglês & EUA & Artigo Original \\
\hline Byron et al. ${ }^{(23)}$ & 2015 & Inglês & EUA & Artigo Original \\
\hline Chesin et al. ${ }^{(24)}$ & 2016 & Inglês & EUA & Artigo de Revisão \\
\hline Cramer et al. ${ }^{(25)}$ & 2016 & Inglês & Alemanha & Artigo de Revisão \\
\hline Frias, Whyne ${ }^{(26)}$ & 2015 & Inglês & EUA & Artigo Original \\
\hline de Vibe et al.(27) & 2013 & Inglês & Noruega & Artigo Original \\
\hline Desrosiersa et al. ${ }^{(28)}$ & 2013 & Inglês & EUA & Artigo Original \\
\hline Di Benedettoa, Swadlingb ${ }^{(29)}$ & 2013 & Inglês & Austrália & Artigo Original \\
\hline Dobie et al. ${ }^{(30)}$ & 2016 & Inglês & Austrália & Artigo Original \\
\hline Dos Santos et al. ${ }^{(31)}$ & 2016 & Inglês & Brasil & Artigo Original \\
\hline Galante et al. ${ }^{(32)}$ & 2017 & Inglês & Reino Unido & Artigo Original \\
\hline Gawrysiak et al. ${ }^{(33)}$ & 2016 & Inglês & EUA & Artigo Original \\
\hline Gerzina, Porfeli ${ }^{(34)}$ & 2012 & Inglês & EUA & Artigo Original \\
\hline Harker et al. ${ }^{\left({ }^{35}\right)}$ & 2016 & Inglês & Austrália & Artigo Original \\
\hline Hazlett-Stevens( $^{(36)}$ & 2012 & Inglês & EUA & Relato de Caso \\
\hline Hjeltnes et al. ${ }^{(37)}$ & 2016 & Inglês & Noruega & Artigo Original \\
\hline Hoge et al..$^{(38)}$ & 2016 & Inglês & EUA & Artigo Original \\
\hline Hosseinzad, Hosseinalipour ${ }^{(39)}$ & 2014 & Inglês & Turquia & Artigo Original \\
\hline Jacobsen et al. ${ }^{(40)}$ & 2011 & Inglês & Reino Unido & Artigo Original \\
\hline Kang et al..$^{(41)}$ & 2008 & Inglês & Coreia & Artigo Original \\
\hline Lacaille et al. ${ }^{(42)}$ & 2017 & Inglês & Canadá & Artigo Original \\
\hline Lilja et al. ${ }^{(43)}$ & 2016 & Inglês & Suécia & Artigo Original \\
\hline López-Navarro et al. . $^{(4))}$ & 2015 & Inglês & Reino Unido & Artigo Original \\
\hline Mitchel, Heads ${ }^{(45)}$ & 2015 & Inglês & EUA & Artigo Original \\
\hline Oken et al. ${ }^{(46)}$ & 2018 & Inglês & EUA & Artigo Original \\
\hline Radford et al. ${ }^{(47)}$ & 2014 & Inglês & Reino Unido & Artigo Original \\
\hline Shorey et al. ${ }^{(48)}$ & 2014 & Inglês & EUA & Artigo Original \\
\hline Spijkerman et al.(49) & 2016 & Inglês & Holanda & Metanálise \\
\hline Tabaka et al. ${ }^{\left({ }^{0}\right)}$ & 2015 & Inglês & EUA & Artigo Original \\
\hline
\end{tabular}

Figura 2 - Caracteristicas gerais dos estudos incluídos $(n=37)$. Santo Antônio de Jesus, Bahia, Brasil, dezembro 2019 
A intervenção mais conhecida dentre as MBIs é o programa de Redução de Estresse Baseado em Mindfulness (MBSR), que acontece em oito sessões semanais com duração de duas horas para se praticar a atenção plena por meio de exercícios de meditação, psicoeducação e discussões em grupo, além de um dia inteiro em um retiro silencioso entre a sexta e a sétima semana $^{(42)}$. Universitários ${ }^{(14,18,37,41)}$ e profissionais da área da saúde(23,30) foram os maiores públicos alvos dessa intervenção dentre os artigos analisados.

Ainda nesse contexto, há a Terapia Cognitiva Baseada em Mindfulness (MBCT), na qual os indivíduos praticantes da MBCT são ensinados a reconhecer e desengatar da ruminação dos pensamentos, tornando-se conscientes deles e de seus sentimentos de forma a não os hipervalorizar e, assim, prevenir e diminuir o pensamento depressivo e possíveis recaídas(43).

Ao se considerar a relação entre o comprometimento às práticas de mindfulness e seus resultados, discute-se a eficácia de tratamentos baseados na atenção plena quando realizados via internet, outro tipo de MBI. Apesar das adaptações, da redução nos sintomas de ansiedade, depressão e insônia, e melhora moderada na qualidade de vida, a adesão continua sendo uma limitação do tratamento(20).

A relação entre o engajamento da prática do mindfulness e seus resultados pode ser associado a menor sofrimento emocional e um estado mental mais positivo dentre os usuários ${ }^{(39)}$ de substâncias psicoativas (SPAs) que integram outro tipo de intervenção encontrada nos artigos, a Prevenção à Recaída Baseada em Mindfulness (MBRP).

A prática das intervenções baseadas em mindfulness proporcionou reduções significantes nos sintomas de ansiedade, aumento no bem-estar e na qualidade de vida(41,45). Percepção mais atenta sobre as experiências internas e externas, melhora na atenção e maior consciência das atividades diárias foram outras habilidades adquiridas ${ }^{(31,42)}$.

Em relação à prática das intervenções baseadas em mindfulness para pessoas com depressão, as informações são conflitantes, pois apesar de não serem encontradas mudanças significativas no escore ${ }^{(41)}$ deste TM, há evidências de remissão(36) dos sintomas de ansiedade e depressão. No que se refere à depressão recorrente, a prática regular de mindfulness pode diminuir os sintomas residuais da depressão(47). Ademais, ainda dentro de transtornos depressivos, cabe trazer que as MBIs podem reduzir o risco da ideação suicida ou a severidade desta em indivíduos com depressão severa(22), inclusive aqueles com comportamento suicida prévio(24).

No que diz respeito ao estresse psicológico, as práticas de intervenções baseadas em mindfulness influenciaram positivamente no bem-estar e na qualidade de vida dos praticantes, aumentando a aceitação, as capacidades observacionais e a autocompaixão, além de diminuir o estresse percebido(16,31). Também possibilitaram maior consciência e controle do estresse, bem como o desenvolvimento de habilidades para manejá-lo(14). Em contrapartida, outro estudo(18) não encontrou redução estatisticamente significante nos níveis de estresse, apesar de apresentar o aumento da aceitação e melhora nas capacidades observacionais. Assim, a atenção plena foi considerada apenas um possível fator de proteção para o estresse ${ }^{(18,26)}$.

Quanto a pessoas com sintomas psicóticos, a prática das intervenções baseadas em mindfulness propiciou a redução de sintomas de ansiedade e depressão e aliviou os sintomas negativos de pessoas com esquizotipia, por tratar-se de uma prática que desenvolve a consciência centrada no presente(15). Em contrapartida, não foi encontrada relação entre mindfulness e sintomas negativos da esquizofrenia, mas a prática está associada à diminuição de comportamentos inibitórios, maior adaptabilidade da regulação emocional e facilidade em lidar com atitudes disfuncionais em pessoas que convivem com esta doença(50). Em uma enfermaria para indivíduos com psicose crônica e resistente ao tratamento, as práticas de mindfulness foram percebidas como uma terapia relaxante, confortável e acessível, sendo considerada aceitável neste ambiente(40).

Há evidências sobre as práticas de mindfulness no cuidado às pessoas com transtornos relacionados ao uso de SPAs como uma prática possivelmente efetiva no manejo das consequências deste uso(48) e dos sintomas de abstinência(21). A habilidade de manter a atenção na experiência imediata, e de aceitação em relação às experiências vividas, são os principais pontos benéficos trabalhados nesta prática, pois compreendem o processo de aprendizagem associativa, desenvolvendo uma crítica sobre a fissura para o uso das SPAs ${ }^{(21)}$. A partir disso, a mudança consciente relacionada ao desejo a partir do não julgamento propiciaria mudanças concretas e eficazes no uso abusivo das substâncias.

A prática de intervenções baseadas em mindfulness também foi observada no tratar da Síndrome de Burnout ${ }^{(17,29,33,35)}$ percebida entre os públicos alvos estudados pelos artigos sobre o mindfulness e ambiente laboral incluídos nesta revisão: médicos residentes em psiquiatria(17); equipe de uma enfermaria de saúde mental para adolescentes ${ }^{(23)}$; psicólogos(29); equipe multidisciplinar de saúde(30); enfermeiros ${ }^{(31)}$; e profissionais de serviço social(35).

Menores níveis de burnout estiveram associados à prática de intervenções baseadas em mindfulness ${ }^{(29,31,34,39)}$ e melhora na capacidade de comunicação, maior conscientização de suas ações, 
e maior conscientização dos padrões emocionais e cognitivos foram relatados após as práticas ${ }^{(17,23,30-31)}$. A empatia foi outra habilidade desenvolvida a partir de práticas de mindfulness que recebeu destaque ${ }^{(17,23)}$, sendo relacionada a menores níveis de burnout, a um melhor relacionamento com colegas de trabalho e maior atenção com os pacientes ${ }^{(17,23)}$. Além disso houve aumento das habilidades de escuta(17); maior capacidade de foco nos momentos das consultas ${ }^{(17,23)}$; e melhora na capacidade de comunicar experiências internas por meio das palavras ${ }^{(31)}$.

Uma vez que interpretações de eventos cotidianos são afetadas por meio da prática da atenção plena e do desenvolvimento do pensamento não-julgador, identificou-se que a flexibilidade mental e a capacidade de ver pensamentos e emoções angustiantes como eventos passageiros, reduz o impacto negativo de eventos estressantes ${ }^{(19)}$. A nível fisiológico, os efeitos percebidos estão relacionados à diminuição dos níveis de cortisol(26), aumento da atividade da telomerase, e mantenimento da pressão arterial e frequência cardíaca em níveis saudáveis ${ }^{(19)}$.

Outros aspectos percebidos e que exprimem relação com a qualidade de vida e bem-estar são a autonomia(21,23,31), a capacidade de se acalmar apenas com as técnicas de mindfulness ${ }^{(16,31)}$ e autocuidado(18,31). A relação entre a prática de mindfulness e o sentir-se capaz, esteve atrelada a relatos sobre como a prática promove o estímulo para que se tornem autocuradores ativos e agentes de mudança ${ }^{(37)}$, bem como a melhora da saúde psicológica por meio do autocuidado(18). Por fim, para além das alterações a nível pessoal, o mindfulness pode aparecer como ferramenta moduladora de interação social(37).

\section{Discussão}

Para que Intervenções Baseadas em Mindfulness (MBI) sejam assim consideradas, é necessário que se baseiem individual ou separadamente na observação do corpo, das sensações, da mente e de atitudes mentais( ${ }^{(51)}$, fundamentos da atenção plena explicados no Satipatthana Sutta(52). A técnica mais utilizada nos estudos analisados, Redução de estresse Baseado em Mindfulness (MBSR) é aplicada como prevenção(53) e atenuação(54) do esgotamento físico ou emocional em indivíduos saudáveis, e àqueles sujeitos a enfrentarem episódios de estresses, respectivamente.

Em relação aos quadros depressivos e recaídas de depressão, é por meio do encorajamento dos participantes a focar em detalhes sobre o ambiente em seu entorno e aceitar as experiências sensitivas que surgirem, sem julgamentos e sem evitá-las, que a Terapia Cognitiva Baseada em Mindfulness (MBCT) refina a conscientização e a retirada do pensamento negativo repetitivo(55), um dos sintomas presentes em quadros de depressão(56).

No tratar de indivíduos acometidos por depressão, porém, a viabilidade e eficácia devem considerar a dificuldade desses indivíduos na adesão às sessões das intervenções baseadas em mindfulness ${ }^{(24)}$. Dessa forma, para um melhor resultado de seus efeitos, a escolha do momento apropriado para sua prática faz-se importante ${ }^{(14)}$, uma vez que, maiores benefícios são percebidos a partir do maior engajamento com as sessões ${ }^{(18)}$. Isso ocorre porque quanto maior o tempo de dedicação à meditação, maiores as chances de resultados na capacidade da aplicação da atenção plena a eventos diários e, por consequência, melhores benefícios psicológicos ${ }^{(42)}$.

A relação entre engajamento e resultados mostra-se importante também na Prevenção à Recaída Baseada em Mindfulness (MBRP), abordagem voltada a usuários de substâncias psicoativas (SPAs) que integra os princípios do MBSR e MBCT(57). Nessa MBI trabalha-se a conscientização sobre sinais de alerta precoces de recaídas, o reconhecimento de gatilhos e reações internas associadas ao uso de SPAs, o desenvolvimento de habilidades eficazes de enfrentamento e o estímulo a escolhas comportamentais focadas na aceitação e tolerância(57). O cultivo da atenção plena pode ser associado a menor sofrimento emocional e um estado mental mais positivo dentre os usuários ${ }^{(39)}$ de SPAs. Assim, objetiva-se diminuir o ímpeto para aliviar o desconforto associado ao uso, promovendo a prevenção à recaída e estimula-se relações interpessoais que reforcem essa rede de proteção(58).

No contexto das intervenções baseadas em mindfulness aplicado aos TM, o gerenciamento do estresse, a redução do grau de ansiedade e o desenvolvimento do foco podem ser conseguidos por meio das MBIs ${ }^{(59)}$. Apesar disso, recomenda-se cautela ao indicar a prática do mindfulness para indivíduos com transtornos de ansiedade, visto que a técnica mal aplicada pode, por exemplo, gerar mais ansiedade em uma pessoa já ansiosa, dificultando a terapêutica(59). Estimular que o indivíduo não crie expectativas em relação aos resultados de cada meditação, contribui para uma melhor prática e maior motivação do sujeito(60).

Além da redução dos sintomas residuais de depressão(47), também percebeu-se que quanto maior o risco de recaída, maior tende a ser a eficácia da prática de $\mathrm{MBCT}^{(61)}$. Esta prática pode ser tão eficaz quanto o uso de medicamentos antidepressivos, em um período de dois anos(61), pois suas práticas auxiliam na redução dos sintomas, no aumento da qualidade de vida e no nível de atenção(43), uma vez que possibilita a compreensão do momento de recaída da doença estimulando a autonomia de sair dele com ajuda das 
técnicas de $\mathrm{MBCT}^{(62)}$. Ademais, a prática vem sendo considerada efetiva na redução da ideação suicida(63-64).

A eficácia das intervenções baseadas em mindfulness foi classificada como moderada para a diminuição de sintomas negativos, sintomas afetivos e melhora da qualidade de vida de pacientes psicóticos ${ }^{(65)}$. Estas práticas podem ser consideradas uma estratégia segura e efetiva na diminuição nos níveis de depressão, na melhora na capacidade de observar as emoções e de agir com consciência para pessoas com psicose precoce $^{(66)}$, que se sentiram mais calmas, acharam uma experiência agradável e relataram melhora acerca da percepção de conviver com um TM(67). Em geral, é interessante ressaltar que as intervenções baseadas em mindfulness podem auxiliar na redução do sofrimento associado a sintomas psicóticos, à medida que auxilia na percepção das emoções e na abertura a experiências internas ${ }^{(68)}$.

Em relação aos transtornos mentais relacionados ao uso de SPAs, as MBIs podem auxiliar no controle cognitivo em situações de vulnerabilidade emocional, ajudando o indivíduo a lidar com esses momentos e interromper comportamentos automáticos. A maior capacidade de descrever experiências emocionais; a adaptação emocional implícita e a resposta emocional autorreguladora proporcionada pela prática das intervenções baseadas em mindfulness são os responsáveis pela diminuição da fissura e dos estados emocionais negativos nesses pacientes ${ }^{(69)}$.

No geral, a abordagem terapêutica por meio do mindfulness é considerada vantajosa em relação a outras abordagens por apresentar impacto moderado para ansiedade e sintomas depressivos; e alto para os níveis de desejo percebido, afetividade negativa e sintomas pós-traumáticos(70).

Sobre as MBIs e o ambiente de trabalho, cabe considerar que o contexto trabalhista cada vez mais precarizado, cercado por um quadro de competição crescente e inseguranças para o trabalhador ${ }^{(71)}$, causa impactos em sua saúde e influencia em seu bem-estar e produtividade, resultando em adoecimento físico e mental e, ciclicamente, maior precarização do ambiente laboral(72). As MBIs, nesse âmbito, tornam-se ferramenta para a melhora da qualidade de vida e bem-estar do trabalhador(73).

No caso da Síndrome de Burnout, que relaciona-se à exposição crônica a fatores estressantes do ambiente laboral sendo composta pelas dimensões da exaustão emocional (EE), despersonalização (DE) e baixa realização profissional $(B R P)^{(74)}$ de forma a afetar a saúde do trabalhador que lida com o cuidado direto a sujeitos(75-76), as MBIs e autocompaixão são sugeridas como práticas eficazes na sua prevenção(29,34-35), visto que trabalham com a diminuição do estresse percebido e da tensão(77). Além da melhora nas habilidades sociais relacionadas à comunicação e relações interpessoais ${ }^{(78)}$, essenciais à assistência e ao cuidado(79-80) e, além disso, são exigências dos espaços de trabalho(81).

Em relação à produtividade, as MBIs auxiliam no aumento da dedicação ao trabalho, na diminuição da rotatividade de empregos e da distração, no aumento do desempenho dentro deste espaço e auxilia na regulação emocional(73).

Os benefícios do mindfulness, enfim, versam sobre os efeitos positivos dessa prática em esferas fisiológicas, psicoemocionais, sociais, físicas e/ou ambientais.Os níveis de cortisol basal diminuíram de forma linear e significativa após MBIs(82-83), e a capacidade de manter a atividade da telomerase foi aumentada por meio da prática da meditação, o que leva a indivíduos vivenciando menos estresse, ansiedade e depressão( ${ }^{(84)}$.

A partir da metodologia da atenção plena aos comportamentos e sensações corporais, pode-se amplificar e fortalecer conexões entre estímulos sensoriais corporais e processos mentais ${ }^{(85)}$. Por meio da meditação focada em seus próprios batimentos cardíacos, por exemplo, resultados de ressonância magnética indicam atividade aprimorada nos córtices insular, somatomotor e cingulado(86). Tais alterações a níveis corticais a partir da prática de mindfulness são possivelmente responsáveis pela melhora das funções cerebrais ligadas à atenção, memória, função executiva, velocidade de processamento e cognição geral(19,87).

Em estudos realizados com idosos, esses efeitos positivos em domínios cognitivos configuram fator de melhor saúde emocional ${ }^{(19,26)}$, uma vez que a maior consciência de suas ações interfere na saúde mental e física(88). Dessa forma, a prática de mindfulness é indicada como positivamente relacionada à vitalidade, satisfação e qualidade de relacionamento consigo mesmo e interpessoal(89).

Por fim, o uso das práticas de MBIs como moduladoras de interação social é possível, porque esta prática auxilia a desenvolver uma consciência com foco no presente, onde a presença física de outras pessoas pode ser suficiente para provocar uma maior atenção social e possibilitar o desenvolvimento da empatia e de tomada de decisões sociais simples ${ }^{(90-91)}$. Além disso, vale ressaltar a importância da rede social fortalecida em casos de MBIs voltadas a usuários de substâncias, uma vez que a reinserção social envolve comportamentos de aceitação e tolerância internas e externas que configuram um novo estilo de vida, prevenindo a recaída frente a situações diárias enfrentadas pelos sujeitos ${ }^{(58)}$.

\section{Considerações Finais}

As práticas de intervenções baseadas em mindfulness têm sido empregadas terapeuticamente em 
adultos e idosos acometidos por transtornos mentais enquanto programas específicos de propósitos distintos. A Redução de Estresse Baseado em Mindfulness (MBSR), a Terapia Cognitiva Baseada em Mindfulness (MBCT) e a Prevenção à Recaída Baseada em Mindfulnes (MBRP) foram os programas mais utilizados nos artigos analisados. Os resultados das MBIs revelaram-se dependentes das variações nas finalidades de cada técnica e nas dimensões das práticas, sendo necessária atenção à população em que será utilizada a técnica, o contexto e os objetivos a serem atingidos. Deve-se ter ressalvas na utilização de práticas de mindfulness via internet, pois, apesar de alguns estudos apresentarem benefícios sobre estas, existem limitações quanto à adesão ao processo terapêutico, além de entraves em relação a orientação terapêutica e interação social, que são pouco efetivas via internet.

As MBIs evidenciaram efeitos e resultados promissores no público adulto e idoso, como a associação desta com a diminuição de sintomas de ansiedade e depressão, além de serem eficazes na prevenção de recaídas e no controle das emoções; a diminuição do risco de ideação suicida em pacientes com depressão severa; e a redução de sintomas de ansiedade e depressão em indivíduos com quadros psicóticos. Para estes últimos, a melhora nos sintomas negativos e nos afetos não foi unânime nos estudos, mas, ainda assim, a prática trouxe benefícios aos pacientes, sobretudo em lidar com as emoções. Além disso, nos ambientes de trabalho, menores níveis de burnout foram associados à prática de MBIs, podendo ser considerados efetivos, inclusive, na prevenção da síndrome e no desenvolvimento de habilidades que resultem em melhorias no ambiente de trabalho, da produtividade e do bem-estar do indivíduo.

Por fim, deve-se ter prudência para a aplicação de qualquer MBI e atentar-se para especificidades de cada população. É importante observar como o sujeito interpreta a prática do mindfulness em sua vida, sua disponibilidade, motivação, engajamento, bem como a criação de expectativas e a ansiedade desenvolvida sobre as práticas e seus resultados.

\section{Referências}

1. Kabat-Zinn J, Massion AO; Kristeller J; Peterson LG; Fletcher KE; Pbet L; Lenderking WR; Santorelli SF. Effectiveness of a Meditation Based Stress Reduction Program in the Treatment of Anxiety Desorders. Am J Psychiatry. 1992;149: 936-43. doi: 10.1176/ ajp.149.7.936.

2. Kabat-Zinn J. Full Catastrophe Living: Using the Wisdom of your Mind to Face Estresse. Pain and Illness. [Kindle Version]. New York: Dell Publishing; 1990.

3. Germer CK. Mindfulness: o que é? Qual a sua importância? In: Germer CK, Siegel RD, Fulton P.
Mindfulness e Psicoterapia. 2. ed. Porto Alegre: Artmed; 2016. p. 19-46.

4. Keng SL, Smoski MJ, Robins CJ. Effects of Mindfulness on Psychological Health: A Review of Empirical Studies. Clin Psychol Rev. 2011; 31(6): 1041-56. doi: 10.1016/j. cpr.2011.04.006.

5. Hölzel BK, Carmody J, Vangel M, Congleton C, Yerramsetti SM, Gard $T$ et al. Mindfulness practice leads to increases in regional brain gray matter density. Psychiatry Res. 2011; 191(1): 36-43. doi: 10.1016/j. pscychresns.2010.08.006.

6. Witkiewitz K, Bowen S, Douglas H, Hsucl SH. Mindfulness-Based Relapse Prevention for Substance Craving. Addict Behav. 2013; 38(2). doi: 10.1016/j. addbeh.2012.04.001.

7. James SL, Abate D, Abate KH, Abay SM, Abbafati C, Abbasi $N$, et al. Global, regional, and national incidence, prevalence, and years lived with disability for 354 diseases and injuries for 195 countries and territories, 1990-2017: a systematic analysis for the Global Burden of Disease Study 2017. Lancet. 2018 Nov; 392(10159): 1789-858. doi: 10.1016/S0140-6736(18)32279-7.

8. World Health Organization. Depression and Other Common Mental Disorders: Global Health Estimates [internet]. Geneva: World Health Organization; 2017. p. 24. [acesso em 18 nov 2019]. Disponível em: https:// apps.who.int/iris/bitstream/handle/10665/254610/ WHO-MSD-MER-2017.2-eng.pdf.

9. Souza MT, Silva MD, Carvalho R. Integrative review: what is it? How to do it? Einstein. 2010; 8(1): 102-6. doi: 10.1590/s1679-45082010rw1134.

10. Botelho L, Cunha C, Macedo M. O método da revisão integrativa nos estudos organizacionais. Gestão e Sociedade. 2011 dez; 5(11):121-36. doi: 10.21171/ ges.v5i11.1220.

11. Karino ME, Felli VEA. Enfermagem Baseada em Evidências: avanços e inovações em revisões sistemáticas. Ciênc Cuid Saúde. 2012; 11:11-5. doi: 10.4025/cienccuidsaude.v11i5.17048.

12. Moher D, Liberati A, Tetzlaff J, Altman DG, PRISMA Group. Preferred reporting items for systematic reviews and meta-analyses: the PRISMA statement. PLoS Med. 2009 Jul 21; 6(7):e1000097. doi: 10.1371/journal. pmed.1000097.

13. Melnyk BM, Fineout-overholt E. Evidence-based practice in nursing and health care: a guide to best practice. Philadelphia: Lippincott; 2011. p. 577.

14. Aherne D, Farrant K, Hickey L, Hickey E, McGrath $L$, McGrath $D$. Mindfulness based stress reduction for medical students: optimising student satisfaction and engagement. BMC Med Educ. 2016 Ago; 16(1):209. doi: 10.1186/s12909-016-0728-8.

15. Antonova E, Amaratunga K, Wright B, Ettinger U, Kumari V. Schizotypy and mindfulness: Magical thinking 
without suspiciousness characterizes mindfulness meditators. Schizophr Res Cogn. 2016 May; 5:1-6. doi: 10.1016/j.scog.2016.05.001.

16. Beck AR, Verticchio $H$, Seeman S, Milliken $E$, Schaab H. A Mindfulness Practice for Communication Sciences and Disorders Undergraduate and SpeechLanguage Pathology Graduate Students: Effects on Stress, Self-Compassion, and Perfectionism. Am J Speech Lang Pathol. 2017 Ago; 26(3):893-907. doi: 10.1044/2017_AJSLP-16-0172.

17. Bentley PG, Kaplan SG, Mokonogho J. Relational Mindfulness for Psychiatry Residents: a Pilot Course in Empathy Development and Burnout Prevention. Acad Psychiatry. 2018 Out; 42(5):668-73. doi: 10.1007/ s40596-018-0914-6.

18. Bergen-Cico D, Possemato K, Cheon S. Examining the efficacy of a brief mindfulness-based stress reduction (Brief MBSR) program on psychological health. J Am Coll Health. 2013; 61(6): 348-60. doi: 10.1080/07448481.2013.813853.

19. Berk L, Warmenhoven F, van Os J, van Boxtel M. Mindfulness Training for People With Dementia and Their Caregivers: Rationale, Current Research, and Future Directions. Front Psychol. 2018 Jun; 9:982. doi: 10.3389/fpsyg.2018.00982.

20. Boettcher J, Aström V, Påhlsson D, Schenström O, Andersson G, Carlbring P. Internet-based mindfulness treatment for anxiety disorders: a randomized controlled trial. Behav Ther. 2014 Mar; 45(2):241-53. doi: 10.1016/j.beth.2013.11.003.

21. Brewer JA, Elwafi HM, Davis JH. Craving to Quit: psychological models and neurobiological mechanisms of mindfulness training as treatment for addictions. Psychol Addict Behav. 2013 Jun; 27(2):366-79. doi: 10.1037/ a0028490.

22. Buitron V, Hill RM, Pettit JW. Mindfulness Moderates the Association Between Perceived Burdensomeness and Suicide Ideation in Adults With Elevated Depressive Symptoms. Suicide Life Threat Behav. 2017 Oct; 47(5):580-88. doi: 10.1111/sltb.12314.

23. Byron G, Ziedonis DM, McGrath C, Frazier JA, deTorrijos F, Fulwiler C. Implementation of Mindfulness Training for Mental Health Staff: Organizational Context and Stakeholder Perspectives. Mindfulness. (NY) 2015 Ago; 6(4):861-72. doi: 10.1007/s12671-014-0330-2.

24. Chesin $M$, Interian $A$, Kline $A$, Benjamin-Phillips C, Latorre M, Stanley B. Reviewing MindfulnessBased Interventions for Suicidal Behavior. Arch Suicide Res. 2016 Oct-Dec; 20(4):507-27. doi: 10.1080/13811118.2016.1162244.

25. Cramer $H$, Lauche $R$, Haller $H$, Langhorst J, Dobos G. Mindfulness- and Acceptance-based Interventions for Psychosis: A Systematic Review and Meta-analysis. Glob
Adv Health Med. 2016 Jan; 5(1): 30-43. doi: 10.7453/ gahmj.2015.083.

26. Frias CM, Whyne E. Stress on health-related quality of life in older adults: the protective nature of mindfulness. Aging Ment Health. 2015; 19(3): 201-6. doi: 10.1080/13607863.2014.924090.

27. de Vibe M, Solhaug I, Tyssen R, Friborg O, Rosenvinge $\mathrm{JH}$, Sørlie T, Bjørndal A. Mindfulness training for stress management: a randomised controlled study of medical and psychology students. BMC Med Educ. 2013 Ago; 13: 107. doi: 10.1186/1472-6920-13-107. 28. Desrosiersa A, Vineb V, Klemanskib DH, NolenHoeksemab S. Mindfulness and Emotion Regulation in Depression and Anxiety: Common and Distinct Mechanisms of Action. Depress Anxiety. 2013 Jul; 30(7):654-61. doi: 10.1002/da.22124.

29. Di Benedetto M, Swadling M. Burnout in Australian psychologists: correlations with work-setting, mindfulness and self-care behaviours. Psychol Health Med. 2014; 19(6):705-15. doi: 10.1080/13548506.2013.861602.

30. Dobie A, Tucker A, Ferrari M, Rogers JM. Preliminary evaluation of a brief mindfulness-based stress reduction intervention for mental health professionals. Australas Psychiatry. 2016 Feb; 24(1):42-5. doi: 10.1177/1039856215618524.

31. dos Santos TM, Kozasa EH, Carmagnani IS, Tanaka LH, Lacerda SS, Nogueira-Martins LA. Positive Effects of a Stress Reduction Program Based on Mindfulness Meditation in Brazilian Nursing Professionals: Qualitative and Quantitative Evaluation. Explore (NY). 2016 MarApr; 12(2): 90-9. doi: 10.1016/j.explore.2015.12.005. 32. Galante J, Dufour G, Vainre M, Wagner AP, Stochl J, Benton $A$, et al. A mindfulness-based intervention to increase resilience to stress in university students (the Mindful Student Study): a pragmatic randomised controlled trial. Lancet Public Health. 2018 Feb; 3(2): 72-81. doi: 10.1016/S2468-2667(17)30231-1.

33. Gawrysiak Michael J, Leongc SH, Grassettid SN, Waib M, Shoreye RC, Baime MJ. Dimensions of distress tolerance and the moderating effects on mindfulnessbased stress reduction. Anxiety Stress Coping. 2016 Sep; 29(5): 552-60. doi: 10.1080/10615806.2015.1085513. 34. Gerzina HA, Porfeli EJ. Mindfulness as a predictor of positive reappraisal and burnout in standardized patients. Teach Learn Med. 2012; 24(4): 309-14. doi: 10.1080/10401334.2012.715255.

35. Harker R, Pidgeon AM, Klaassen F, King S. Exploring resilience and mindfulness as preventative factors for psychological distress burnout and secondary traumatic stress among human service professionals. Work. 2016 Jun 8 ; 54(3): 631-7. doi: 10.3233/WOR-162311.

36. Hazlett-Stevens $H$. Mindfulness-based stress reduction for comorbid anxiety and depression: case report and clinical considerations. J Nerv Ment 
Dis. 2012 Nov; 200(11): 999-1003. doi: 10.1097/ NMD.0b013e3182718a61.

37. Hjeltnes A, Moltu C, Schanche E, Jansen Y, Binder PE. Both sides of the story: Exploring how improved and less-improved participants experience mindfulnessbased stress reduction for social anxiety disorder. Psychother Res. 2018 Jan; 28(1): 106-122. doi: 10.1080/10503307.2016.1169330.

38. Hoge EA, Guidos BM, Mete M, Bui E, Pollack MH, Simon NM. Effects of mindfulness meditation on occupational functioning and health care utilization in individuals with anxiety. J Psychosom Res. 2017 Apr; 95: 7-11. doi: 10.1016/j.jpsychores.2017.01.011.

39. Hosseinzadeh AsI NR, Hosseinalipour F. Effectiveness of Mindfulness-Based Stress Reduction Intervention for Health-Related Quality of Life in Drug-Dependent Males. Iran Red Crescent Med J. 2014 Sep; 16(9): e12608. doi: $10.5812 / \mathrm{ircmj} .12608$.

40. Jacobsen P, Morris E, Johns L. Mindfulness Groups for Psychosis; Key Issues for Implementation on an Inpatient Unit. Behav Cogn Psychother. 2011; 39: 34953. doi: $10.1017 /$ S1352465810000639.

41. Kang YS, Choi SY, Ryu E. The effectiveness of a stress coping program based on mindfulness meditation on the stress, anxiety, and depression experienced by nursing students in Korea. Nurse Educ Today. 2009 Jul; 29(5): 538-43. doi: 10.1016/j.nedt.2008.12.003.

42. Lacaille J, Sadikaj G, Nishioka M, Carrière K, Flanders J, Knäuper B. Daily Mindful Responding Mediates the Effect of Meditation Practice on Stress and Mood: The Role of Practice Duration and Adherence. J Clin Psychol. 2018 Jan; 74(1): 109-22. doi: 10.1002/jclp.22489.

43. Lilja JL, Zelleroth C, Axberg U, Norlander T. Mindfulness-based cognitive therapy is effective as relapse prevention for patients with recurrent depression in Scandinavian primary health care. Scand J Psychol. 2016 Oct; 57(5): 464-72. doi: 10.1111/sjop.12302.

44. López-Navarro E, Del Canto C, Belber M, Mayol A, Fernández-Alonso $O$, Lluis J. Mindfulness improves psychological quality of life in community-based patients with severe mental health problems: A pilot randomized clinical trial. Schizophr Res. 2015 Oct; 168(1-2):530-6. doi: 10.1016/j.schres.2015.08.016.

45. Mitchell M, Heads G. Staying Well: A Follow Up of a 5-Week Mindfulness Based Stress Reduction Programme for a Range of Psychological Issues. Community Ment Health J. 2015 Nov; 51(8):897-902. doi: 10.1007/ s10597-014-9825-5.

46. Oken BS, Goodrich E, Klee D, Memmott T, Proulx J. Predictors of Improvements in Mental Health From Mindfulness Meditation in Stressed Older Adults. Altern Ther Health Med. [Internet]. 2018 Jan [cited Dec 27 2019] ; 24(1):48-55. Available from: https://www.ncbi. nlm.nih.gov/pubmed/29332020.
47. Radford S, Eames C, Brennan K, Lambert G, Crane C, Williams JM. Trait mindfulness as a limiting factor for residual depressive symptoms: an explorative study using quantile regression. PLoS One. 2014 Jul; 9(7):e100022. doi: 10.1371/journal.pone.0100022.

48. Shorey R, Brasfield H, Anderson S, Stuart GL. Differences in Trait Mindfulness across Mental Health Symptoms among Adults in Substance Abuse Treatment. Subst Use Misuse. 2014 Apr; 49(5):595-600. doi: 10.3109/10826084.2014.850310.

49. Spijkerman MP, Pots WT, Bohlmeijer ET2. Effectiveness of online mindfulness-based interventions in improving mental health: A review and meta-analysis of randomised controlled trials. Clin Psychol Rev. 2016 Apr; 45:102-14. doi: 10.1016/j.cpr.2016.03.009.

50. Tabak NT, Horanb WP, Green MF. Mindfulness in schizophrenia: Associations with self-reported motivation, emotion regulation, dysfunctional attitudes, and negative symptoms. Schizophr Res. 2015 Oct; 168(0):537-42. doi: 10.1016/j.schres.2015.07.030.

51. Coutiño AM. Terapias cognitivo-conductuales de tercera generación ( $\mathrm{ttg}$ ): la atención plena/ mindfulness. Rev Int Psicol. 2012 Jul; 12(01). doi: 10.33670/18181023.v12i01.66.

52. Nikaya $M$ [homepage na internet]. Satipatthana Sutta: El Sutra de los fundamentos de la atención [Acceso 20 dic 2019]. Disponíble en: http://www. librosbudistas.com/descargas/SATI.htm.

53. Khoury B, Sharma M, Rush SE, Fournier C. Mindfulness-based stress reduction for healthy individuals: A meta-analysis. J. Psychosom. Res. 2015; 78(6):519-28. doi: 10.1016/j.jpsychores.2015.03.009. 54. Song $Y$, Lindquist $R$. Effects of mindfulnessbased stress reduction on depression, anxiety, stress and mindfulness in Korean nursing students. Nurse Educ. Today. 2015; 35(1):86-90. doi: 10.1016/j. nedt.2014.06.010.

55. Gu J, Strauss C, Bond R, Cavanagh K. How do mindfulness-based cognitive therapy and mindfulnessbased stress reduction improve mental health and wellbeing? A systematic review and meta-analysis of mediation studies. Clin Psychol Rev 2015; 37:1-12. doi: 10.1016/j.cpr.2015.01.006.

56. Faccioli JS. Efeitos do pensar sobre o passado: pensamentos contrafactuais de estudantes universitários com e sem indicativos de depressão [tese na internet]. São Carlos: Universidade Federal de São Carlos UFSCAR; 2017 [Acesso $21 \mathrm{dez}$ 2019]. Disponível em: https://repositorio.ufscar.br/handle/ufscar/9393.

57. Bowen S, Chawla N, Collins SE, Witkiewitz K, Hsu S, Grow J, et al. Mindfulness-Based Relapse Prevention for Substance Use Disorders: A Pilot Efficacy Trial. Subst Abus. 2009 Oct-Dec; 30(4):295-305. doi: $10.1080 / 08897070903250084$. 
58. Limberger J, Mello LTN, Schneider JA, Andretta I. Mindfulness no Treinamento em Habilidades Sociais para Usuários de Drogas. Rev Bras Ter Cogn. 2017; 13(2):8491. doi: 10.5935/1808-5687.20170013.

59. Martins MSPP, Seixas PT, Meneses RM, Barreto ESS, Pinto PSP. Mindfulness: um estudo sobre a percepção dos terapeutas cognitivos no público soteropolitano. SEPA - Seminário Estudantil de Produção Acadêmica, UNIFACS; 2017. 16. Disponível em: https://revistas. unifacs.br/index.php/sepa/article/view/5010.

60. Mesquita TO, Furtado TMG. Tratamento da ansiedade através da aplicação de técnicas mindfulness: uma revisão de literatura. Rev Gestão Saúde [Internet]. 2019 [Acesso 27 dez 2019]; 20(1):65-78. Disponível em: http://www.herrero.com.br/files/revista/ file1af9a584827d745aa7a4c571890c2cba.pdf.

61. Kuyken W, Hayes R, Barrett B, Byng R, Dalgleish T, Kessler D, Lewis G, Watkins E, Morant N, Taylor RS, Byford S. The effectiveness and cost-effectiveness of mindfulness-based cognitive therapy compared with maintenance antidepressant treatment in the prevention of depressive relapse/recurrence: results of a randomised controlled trial (the PREVENT study). Health Technol Assess. 2015 Sep; 19(73):1-124. doi: 10.3310/ hta19730.

62. Williams CM, Meeten F, Whiting S. 'I had a sort of epiphany!' An exploratory study of group mindfulnessbased cognitive therapy for older people with depression. Aging Ment Health. 2018 Feb; 22(2):208-17. doi: 10.1080/13607863.2016.1247415.

63. Rizzuti MS. Uso da terapia cognitiva baseada em mindfulness na prevenção de recaída para pacientes com depressão: revisão sistemática. Salvador: Universidade do estado da Bahia (UNEB); 2015 [Acesso 23 dez 2019]. Disponível em: http://www.saberaberto. uneb.br/bitstream/20.500.11896/651/1/TCC\%20 Mindfulness\%20e\%20Depressao.pdf.

64. Forkmann T, Wichers M, Geschwind N, Peeters F, van Os J, Mainz V, Collip D. Effects of mindfulnessbased cognitive therapy on self-reported suicidal ideation: results from a randomised controlled trial in patients with residual depressive symptoms. Compr Psychiatry 2014;55(8):1883-90. doi: 10.1016/j. comppsych.2014.08.043.

65. Khoury B, Lecomte T, Gaudiano BA, Paquin K. Mindfulness interventions for psychosis: A metaanalysis. Schizophr Res 2013; 150(1):176-84. doi: 10.1016/j.schres.2013.07.055.

66. Miró MT, Arceo J, Ibáñez I. Mindfulness en la Psicosis: Un Estudio Piloto. Rdp. 2016; 27(103): 37-56. doi: $10.33898 /$ rdp.v27i103.103.

67. Tong ACY, Lin JJX, Cheung VYK, Lau NKM, Chang WC, Chan SKW, Hui CLM, Lee EHM, Chen EYH. A LowIntensity Mindfulness-Based Intervention for Mood
Symptoms in People with Early Psychosis: Development and Pilot Evaluation. Clin Psychol Psychother .2015; 23(6): 550-60. doi:10.1002/cpp.1981.

68. Carvalho DMH. Processamento da Ameaça na Psicose: o contributo da autoaversão, dos medos da compaixão e do mindfulness. Coimbra: Universidade de Coimbra - Faculdade de Psicologia e de Ciências da Educação UF/FPCE; 2015 [Acesso 29 dez 2019]. Disponível em: https://eg.uc.pt/handle/10316/31693. 69. Henriques ESC. Contributo da Regulação Emocional Implícita para a Eficácia do Treino de Mindfulness na População com Perturbação de Uso de Substâncias. Algarve: Faculdade de Ciências Humanas e Sociais, Universidaded do Algarve; 2018 [ Acesso $29 \mathrm{dez}$ 2019]. Disponível em: https://sapientia.ualg.pt/ handle/10400.1/12647.

70. Cavicchioli M, Movalli M, Maffei C. The Clinical Efficacy of Mindfulness-Based Treatments for Alcohol and Drugs Use Disorders: A Meta-Analytic Review of Randomized and Nonrandomized Controlled Trials. Eur Addict Res .2018; 24(3):137-62. doi:10.1159/000490762.

71. Cardoso AC, Morgado L. trabalho e saúde do trabalhador no contexto atual: ensinamentos da Enquete Europeia sobre Condições de Trabalho. Saúde Soc. 2019;28(1) https://doi.org/10.1590/ S0104-12902019170507.

72. Duarte RF. Os riscos psicossociais no trabalho e as políticas públicas de preservação da saúde mental do trabalhador. Franca: Universidade Estadual Paulista "Júlio De Mesquita Filho"; 2018 [Acesso 29 dez 2019]. Disponível em: https://repositorio.unesp.br/ handle/11449/180559.

73. Markus PMN, Lisboa CSM. Mindfulness e seus beneficios nas atividades de trabalho e no ambiente organizacional. Rev Graduação. [Internet]. 2015 [Acesso 29 dez 2019];8(1). Disponível em: http:// revistaseletronicas.pucrs.br/ojs/index.php/graduacao/ article/view/20733/0.

74. Aguiar CVN, Silva EEC, Carvalho BR, Ferreira JCM, Jesus KCO. Cultura organizacional e adoecimento no trabalho: uma revisão sobre as relações entre cultura, burnout e estresse ocupacional. Rev Psicol Divers Saúde 2017 Maio;6(2):121-31. doi: 10.17267/2317-3394rpds. v6i2.1157.

75. Silveira KA, Enumo SRF, Paula KMP; Batista EP. Estresse e enfrentamento em professores: uma análise da literatura. Educ Rev. [Acesso 29 dez 2019]; 2014;30(4): 15-36. Disponível em: http://www.scielo. br/pdf/edur/v30n4/02.pdf.

76. Picheth CFP, Selow MLC, Toniolo RMM. A gestão de qualidade em profissionais enfermeiros diagnosticados com a síndrome de burnout. Rev Dom Acadêmico. [Internet]. $2016 \mathrm{Jul} / \mathrm{Dez}$ Acesso $29 \mathrm{dez}$ 2019]; 1(1): 89-109. Disponível em: https://domacademico. 
unidombosco.edu.br/index.php/domacademico/article/ viewFile/11/11.

77. Gracia-Gracia P, Oliván-Blázquez B. Burnout and Mindfulness Self-Compassion in Nurses of Intensive Care Units. Holistic Nurs Practice 2017;31(4):225-33. doi: $10.1097 /$ hnp.0000000000000215.

78. Montezeli JH, Almeida KP, Haddad MCFL. Nurses' perceptions about social skills in care management from the perspective of complexity. Rev Esc Enferm USP. 2018;52:e03391. doiI: http://dx.doi.org/10.1590/ S1980-220X2017048103391.

79. Albuquerque MCS, Souza DFS, Maynart WHC, Bezerra LFD, Cassimiro ARTS, Cavalcante JC. Empatia dos profissionais de enfermagem de um serviço hospitalar de emergência. Texto Contexto Enferm. 2019;2 e20170406. doi: http://dx.doi. org/10.1590/1980-265x-tce-2017-0406.

80. Almeida Q, Fófano GA. Tecnologias leves aplicadas ao cuidado de enfermagem na unidade de terapia intensiva: uma revisão de literatura. HU Rev. [Internet]. 2016 set/out [Acesso $29 \mathrm{dez}$ 2019]. ;42(3): 191-6. Disponível em: https://periodicos.ufjf.br/index.php/ hurevista/article/view/2494/891.

81. Galdino CBT, Araujo CF, Cantilino A. Treinamento de Mindfulness aplicado em psicoterapeutas: uma Revisão Narrativa. Rev Bras Terap Cogn. 2014;10(2):65-72. doi: 10.5935/1808-5687.20180009.

82. Carpena MX, Menezes CB. Efeito da Meditação Focada no Estresse e Mindfulness Disposicional em Universitários. Psicologia: Teoria e Pesquisa. 2018. doi: 10.1590/0102.377e3441.

83. Fan Y, Tang YY, Posner MI. Cortisol level modulated by integrative meditation in a dose-dependent fashion. Stress Health. 2014 Feb;30(1):65-70. doi: 10.1002/smi.2497.

84. Alda M, Puebla-Guedea M, Rodero B, Demarzo M, Montero-Marin J, Roca $M$ et al. Zen meditation, Length of Telomeres, and the Role of Experiential Avoidance and Compassion. Mindfulness.2016; 7:651-59. doi: 10.1007/ s12671-016-0500-5.

85. Tang YY. The Neuroscience of Mindfulness Meditation. Cham: Palgrave Macmillan; 2017. p. 23-8. doi; 10.1007/978-3-319-46322-3_3.

86. Critchley HD, Wiens S, Rotshtein P, Ohman A, Dolan RJ. Neural systems supporting interoceptive awareness. Nat Rev Neurosci; 2004; 7:189-95. doi: 10.1038/nn1176. 87. Reis WGP. Evidências do papel de mindfulness no aprimoramento das funções executivas [monografia de internet]. Belo Horizonte: Universidade Federal de Minas
Gerais, Instituto de Ciências Biológicas; 2014 [Acesso 22 dez 2019]. Disponível em: http://hdl.handle.net/1843/ VRNS-9N4FVQ.

88. Silva ACC, Assumpção AA. A influência de mindfulness na qualidade de vida de idosos: revisão narrativa. Pretextos - Psicol Rev. [Internet]; 2018 [Acesso 22 dez 2019]; 3(6):37-51. Disponível em: http://periodicos.pucminas.br/index.php/pretextos/ article/view/18404.

89. Antunes GMC. Mindfulness, stresse, psicopatologia e estratégias para a gestão de conflitos em cuidadores de pessoas com incapacidade intelectual [tese na internet]. Coimbra: Instituto Superior Miguel Torga, Escola Superior de Altos Estudos; 2015 [Acesso $22 \mathrm{dez}$ 2019]. Disponível em: http://repositorio.ismt.pt/jspui/ handle/123456789/592.

90. Thantrey HH. Relação entre mindfulness, pensamentos automáticos e autocompaixão em população adulta. Instituto de Psicologia Aplicada [tese de internet]. ISPA - Instituto Universitário; 2016 [Acesso 23 dez 2019]. Disponível em: http://repositorio.ispa.pt/ handle/10400.12/5106.

91. Van Doesum NJ, Karremans JC, Fikke RC, Lange MA, Van Lange PAM. Social mindfulness in the real world: the physical presence of others induces other-regarding motivation. Soc Influence. 2018; 13(4):209-22. doi: 10.1080/15534510.2018.1544589.

\section{Contribuição dos autores}

Concepção e planejamento do estudo: Paula Hayasi Pinho. Obtenção dos dados: Paula Hayasi Pinho, Lígia Maffei Carnevalli, Renata Oliveira Santos e Luanna Carolyne Silva De Lacerda. Análise e interpretação dos dados: Paula Hayasi Pinho, Lígia Maffei Carnevalli, Renata Oliveira Santos e Luanna Carolyne Silva De Lacerda. Análise estatística: Paula Hayasi Pinho e Luanna Carolyne Silva De Lacerda. Redação do manuscrito: Paula Hayasi Pinho, Lígia Maffei Carnevalli, Renata Oliveira Santos e Luanna Carolyne Silva De Lacerda. Revisão crítica do manuscrito: Paula Hayasi Pinho e Luanna Carolyne Silva De Lacerda.

Todos os autores aprovaram a versão final do texto. Conflito de interesse: os autores declararam que não há conflito de interess

Recebido: 26.01.2020 Aceito: 26.05 .2020 